International Journal of Modern Physics A

Vol. 34, No. 29 (2019) 1950180 (22 pages)

(C) The Author(s)

DOI: $10.1142 /$ S0217751X1950180X

\title{
Equivalence principle for antiparticles and its limitations
}

\author{
U. D. Jentschura \\ Department of Physics, Missouri University of Science and Technology, \\ Rolla, Missouri 65409, USA \\ ulj@mst.edu
}

Received 24 August 2019

Revised 26 September 2019

Accepted 7 October 2019

Published 23 October 2019

\begin{abstract}
We investigate the particle-antiparticle symmetry of the gravitationally coupled Dirac equation, both on the basis of the gravitational central-field problem and in general curved space-time backgrounds. First, we investigate the central-field problem with the help of a Foldy-Wouthuysen transformation. This disentangles the particle from the antiparticle solutions, and leads to a "matching relation" of the inertial and the gravitational mass, which is valid for both particles as well as antiparticles. Second, we supplement this derivation by a general investigation of the behavior of the gravitationally coupled Dirac equation under the discrete symmetry of charge conjugation, which is tantamount to a particle $\rightarrow$ antiparticle transformation. Limitations of the Einstein equivalence principle due to quantum fluctuations are discussed. In quantum mechanics, the question of where and when in the Universe an experiment is being performed can only be answered up to the limitations implied by Heisenberg's Uncertainty Principle, questioning an assumption made in the original formulation of the Einstein equivalence principle. Furthermore, at some level of accuracy, it becomes impossible to separate nongravitational from gravitational experiments, leading to further limitations.
\end{abstract}

Keywords: Dirac equation; general relativity; curved space-time; antiparticles; fundamental symmetries; inertial and gravitation mass; Penrose conjecture; $S O(1,3)$ gauge group.

PACS numbers: 03.65.-w, 04.62.+v, 11.15.Bt, 12.20.Ds

This is an Open Access article published by World Scientific Publishing Company. It is distributed under the terms of the Creative Commons Attribution-NonCommercial-NoDerivatives 4.0 (CC BY-NC-ND) License which permits use, distribution and reproduction, provided that the original work is properly cited, the use is non-commercial and no modifications or adaptations are made. 


\section{Introduction}

A significant motivation for antimatter tests of gravity is that the equivalence principle has never been tested for antiparticles. We aim to give an account of the implications of the gravitationally coupled Dirac equation for antiparticles, motivated by a couple of ongoing experiments at CERN. ${ }^{1-8}$ Here, we investigate, in detail, the relation of the inertial mass of spin-1/2 particles and antiparticles, based on the Dirac equation which, as is well known to atomic theorists ${ }^{9}$ but perhaps less well known in the general relativity community, describes both particles as well as the corresponding antiparticles simultaneously. We observe that, a priori, it is the inertial, not the gravitational, mass which enters the Dirac equation. However, we will attempt to show that, by coupling the Dirac equation to curved space-time, it is possible to identify the relation of the inertial mass to the gravitational mass, for both particles and antiparticles. A number of consequences of our results are discussed.

Dirac is sometimes quoted as saying that the equation named after him ${ }^{10,11}$ is "more intelligent than its inventor." Indeed, the Dirac equation solved a number of problems simultaneously: (i) It provided the necessary linearization of the KleinGordon equation, thus solving problems connected with the negative probabilities otherwise encountered in the context of the Klein-Gordon equation. The linearization also enabled physicists to formulate a Hamiltonian operator for spin- $1 / 2$ particles. $\frac{12}{2}$ (ii) The Dirac equation immediately led to a consistent description of an intrinsic degree of freedom of an elementary particle, namely, the spin of the electron (and also, of the spin of the positron). (iii) The Dirac equation predicted the existence of particles of the same (inertial!) mass, but opposite charge, namely, the existence of spin-1/2 antiparticles. Indeed, the existence of the positron was confirmed after the Dirac equation was invented. $\frac{13}{}$ For an illustrative and interpretive discussion of some interesting properties of the Dirac equation, see Ref. 14.

One might ask: If the Dirac equation makes a definite prediction about the charge of the antiparticles, then why could the Dirac equation not be used in order to make a definitive prediction about the gravitational mass of the antiparticles? One should remember that conceivable gravitational matter-antimatter repulsion has been investigated in a number of theoretical papers (see, e.g. Ref. 15).

The answer is as follows: (i) First, one has to realize that the mass which enters the free Dirac equation (no coupling to either electromagnetic or gravitational fields), constitutes the inertial (not the gravitational) mass of the particle. After a transformation which disentangles the particle from the antiparticle, known as the Foldy-Wouthuysen transformation, ${ }^{16}$ one can derive the energy-momentum relation for both particles as well as antiparticles. One then immediately realizes that the Dirac equation predicts the same inertial mass for both kinds of particles.

(ii) The second step is to couple the Dirac particle to an external electromagnetic field, and carry out the Foldy-Wouthuysen transformation, which in this case becomes a little more involved. After disentangling the particle from the antiparticle 
Hamiltonian, one realizes that the electric charge of the Dirac antiparticle has to be equal in magnitude, but opposite in sign, to that of the particle. In order to address a possible misunderstanding right from the start, let us also remember that, in order to derive antiparticle properties from the Dirac equation, it is not necessary to quantize the Dirac field: Namely, the negative-energy solutions of the Dirac equation are interpreted as antiparticle solutions, in view of the reinterpretation principle. ${ }^{12,17}$ In that sense, there is no such thing as a "single-particle Dirac theory": Rather, the solutions of the equation itself describe particles (positive energy) as well as antiparticles (negative energy). Recall that, historically, the prediction of the existence of the positron (with all of its electromagnetic as well as inertial properties) on the basis of the Dirac equation ${ }^{10,11}$ was followed by the discovery of the positron, $\frac{13}{13}$ long before the concept field-quantization was introduced in theoretical physics. 18

(iii) The third step then is to couple the Dirac equation to a gravitational field. Here, too, a possible misunderstanding needs to be addressed right from the start: One might think that it is necessary to quantize gravity. However, that is not the case. General relativity is a classical theory which promotes space-time to a curved structure; events are described by space-time coordinates. In consequence, the wave function of a particle (in general relativity) becomes a function of the space-time coordinates. $\stackrel{19-24}{2}$ One then has to couple the Dirac particle to the curved spacetime. The foundations of that endeavor have been laid by Tetrode, ${ }^{25}$ Fock and Ivanenko, ${ }^{26-28}$ Weyl, ${ }^{29}$ and Brill and Wheeler ${ }^{30}$ (for an excellent historic account of the early developments, see Ref. 31). One needs to introduce a covariant coupling of the Dirac particles, with respect to changes in local Lorentz frames. ${ }^{27,32}$ These changes in local Lorentz frames (space-time is locally flat) assume the role of a (local) $S O(1,3)$ gauge transformation, which can be formulated for spin- $1 / 2$ particles. ${ }^{27,30,32-34}$ This is analogous to the electromagnetic case, where the gauge transformation is that of the underlying $U(1)$ gauge theory. Expressed differently, the gravitational field, in this case, assumes the same role as a classical, external, electromagnetic field entering the Dirac equation (e.g. an external Coulomb field), and does not need to be quantized.

After applying the Foldy-Wouthuysen transformation to the gravitationally coupled Dirac equation, one obtains the effective particle and antiparticle Hamiltonians. Expressed differently, one starts with a theory in which the mass parameter assumes the role of the inertial mass, for both particles as well as antiparticles, and hopes to end up with two Hamiltonians: the first of these is applicable to the particle, while the second Hamiltonian describes the gravitational coupling of the antiparticle. It is then of interest to analyze in which manner the (inertial) mass of the particle (and antiparticle) enter the final, gravitationally coupled effective particle and antiparticle Hamiltonians, i.e. to investigate the relationship of the gravitational and inertial masses. Under favorable circumstances, one should be able to determine the functional relationship of the inertial mass and the gravitational mass, for both the particle and the antiparticle. 
Again, in order to avoid further possible misunderstandings, we should remember that, quite recently, in very remarkable experiments, the equality of the chargeto-inertial-mass ratio of protons and antiprotons has been experimentally verified to unprecedented precision. $\frac{35,36}{}$ These results, however, do not have any connection to the gravitational mass of the antiparticles, which is the subject of the current investigation.

As a last possible interjection, one might point out that many particles used in fundamental physics experiments on the properties of antimatter, actually constitute composite particles. For example, the mass of antiprotons mostly is the mass equivalent of the extra energy of the quarks and gluons in a region within the antiproton, as compared to the rest energy of the quarks alone in the quantum chromodynamic vacuum, accounts for about $99 \%$ of the mass of the proton. As the free quarks contribute little to the hadron masses, the identical inertial masses of protons and antiprotons are thus due mostly to the color (and electroweak) interactions which have the same strengths between charges as between anticharges (proton versus antiproton). In the context of the current investigation, what matters is that, for purposes of the description of quantum electrodynamic bound states, protons (and antiprotons) can be described to excellent accuracy by an effective Dirac equation which includes form factors (see, e.g. Ref. 37). On the level of the effective Dirac equation, protons and antiprotons are thus amenable to an analysis based on the same formalism as originally devised for point-like spin-1/2 particles (electrons and positrons).

We organize the paper as follows. In Sec. 2, we discuss the derivation of the gravitationally coupled Dirac equation in great detail. The equivalence principle for antimatter is explored in Sec. 3. Conclusions are reserved for Sec. 4. Three appendices round off the paper. Appendix A is devoted to a discussion of the differences between the gravitational and electrostatic central-field problems. Limitations of Einstein's Equivalence Principle (EEP) due to quantum effects are discussed in App. B. The relationship of our investigations to the Penrose conjecture is treated in App. C. Units with $\hbar=\epsilon_{0}=c=1$ are used throughout the paper unless otherwise stated.

\section{Formalism for Gravitational Coupling}

\subsection{Free Dirac equation}

First of all, we should recall the free Dirac equation,,$\underline{10}-12$ which in covariant form reads as

$$
\left(\mathrm{i} \gamma^{\mu} \partial_{\mu}-m_{I}\right) \psi=0 \text {. }
$$

The mass parameter in the free Dirac equation is the inertial mass $m_{I}$ (further remarks on this point are given in the following). Throughout this article, we use the Dirac matrices $\gamma^{\mu}$ in the Dirac representation,

$$
\gamma^{0}=\left(\begin{array}{cc}
\mathbb{1}_{2 \times 2} & 0 \\
0 & -\mathbb{1}_{2 \times 2}
\end{array}\right), \quad \vec{\gamma}=\left(\begin{array}{cc}
0 & \vec{\sigma} \\
-\vec{\sigma} & 0
\end{array}\right),
$$


where the $\vec{\sigma}$ are the Pauli matrices. We can convert this equation into a noncovariant form, and write the free Dirac Hamiltonian $H_{\mathrm{FD}}$ as

$$
H_{\mathrm{FD}}=\vec{\alpha} \cdot \vec{p}+\beta m_{I}, \quad \beta=\gamma^{0}, \quad \vec{\alpha}=\beta \vec{\gamma} .
$$

Let us calculate the square of the noncovariant form of the free Dirac equation $\mathrm{i} \partial_{t} \psi=H_{\mathrm{FD}} \psi$. Using the fact that $\{\vec{\alpha}, \beta\}=0$, one obtains

$$
-\partial_{t}^{2} \psi=\left[\left(\vec{\alpha} \cdot \vec{p}+\beta m_{I}\right)^{2}\right] \psi=\left(\vec{p}^{2}+m_{I}^{2}\right) \psi .
$$

For stationary states, one can replace i $\partial_{t} \rightarrow E$, and thus $-\partial_{t}^{2} \rightarrow E^{2}$. The dispersion relation

$$
E= \pm \sqrt{\vec{p}^{2}+m_{I}^{2}}= \pm\left(m_{I}+\frac{\vec{p}^{2}}{2 m_{I}}-\frac{\vec{p}^{4}}{8 m_{I}^{3}}+\cdots\right)
$$

dictates that $m_{I}$ should be interpreted as the inertial mass, not the gravitational mass. Restoring SI units temporarily, we see that the first term $m_{I}$ is just the restmass energy $m_{I} c^{2}$, while the second term describes the Schrödinger energy, and the third gives the relativistic correction.

In fact, the (unitary) Foldy-Wouthuysen (FW) transformation ${ }^{16}$ brings the free Dirac (FD) equation into diagonal form,

$$
\begin{aligned}
H_{\mathrm{FD}}^{\mathrm{FW}}=U H_{\mathrm{FD}} U^{-1} & =\left(\begin{array}{cc}
\sqrt{\vec{p}^{2}+m_{I}^{2}} \mathbb{1}_{2 \times 2} & 0 \\
0 & -\sqrt{\vec{p}^{2}+m_{I}^{2}} \mathbb{1}_{2 \times 2}
\end{array}\right) \\
& =\left(\begin{array}{cc}
H_{\mathrm{FD}}^{+} & 0 \\
0 & -H_{\mathrm{FD}}^{-}
\end{array}\right),
\end{aligned}
$$

where $\mathbb{1}_{2 \times 2}$ is the two-dimensional unit matrix. Here, $H_{\mathrm{FD}}^{+}$is the $(2 \times 2)$ particle Hamiltonian, while $H_{\mathrm{FD}}^{-}$is the $(2 \times 2)$ antiparticle Hamiltonian. The minus sign is due to the reinterpretation principle ${ }^{38,39}$ for antiparticles, which implies that an antiparticle with eigenvalue $-E$ of the time derivative operator $i \partial_{t}$ is interpreted as an antiparticle state with physical energy $E$.

The transformation $U$ is constructed, in the general case, as

$$
U=\mathrm{e}^{\mathrm{i} S}, \quad S=-\mathrm{i} \beta \frac{\mathcal{O}}{2 m_{I}},
$$

where $\mathcal{O}$ is the "odd" part [in $(2 \times 2)$ bispinor space] of the Dirac Hamiltonian. In general, the elimination of the odd operators is based on the identity

$$
[\beta, \beta \mathcal{O}]=\mathcal{O},
$$

which holds for a general odd matrix $\mathcal{O}$. For the free Dirac Hamiltonian (3), one sets

$$
\mathcal{O}=\vec{\alpha} \cdot \vec{p}
$$


which eliminates the term of first order in the momenta in Eq. (3). The relativistic correction terms are obtained if one uses instead

$$
\begin{aligned}
\mathcal{O} & =\vec{\alpha} \cdot \vec{p} \theta(|\vec{p}|) \approx \vec{\alpha} \cdot \vec{p}-\frac{(\vec{\alpha} \cdot \vec{p})^{3}}{6 m_{I}^{2}}, \\
\theta(|\vec{p}|) & =\frac{1}{2|\vec{p}|} \arctan \left(\frac{|\vec{p}|}{m_{I}}\right) .
\end{aligned}
$$

In summary, one obtains the equivalent free Dirac Hamiltonians $H_{\mathrm{FD}}^{+}$and $H_{\mathrm{FD}}^{-}$for particles and antiparticles,

$$
H_{\mathrm{FD}}^{+}=H_{\mathrm{FD}}^{-}=\sqrt{\vec{p}^{2}+m_{I}^{2}} \mathbb{1}_{2 \times 2} .
$$

The same inertial mass $m_{I}$ enters both $H_{\mathrm{FD}}^{+}$and $H_{\mathrm{FD}}^{-}$.

\subsection{Covariant gravitational coupling}

The theoretical formulation of the gravitational coupling of Dirac particles was developed when scientists were trying to understand the connection of Einstein's general theory of relativity to quantum mechanics ${ }^{25-30}$ (see also Ref. 31); a particularly pointed formulation is the "geometrization" of "Dirac's theory of the electron" as the title of Ref. 27 suggests. The positron was not mentioned in the title of the publication, 27 for obvious reasons: it had not even been discovered yet. 13

Now, let us try to present a derivation of the gravitational coupling terms for antiparticles; we will concentrate on the leading terms, namely, those relevant to the nonrelativistic limit. The gravitational coupling of the Dirac equation entails two replacements as compared to Eq. (1),

$$
\gamma^{\mu} \rightarrow \bar{\gamma}^{\mu}, \quad \partial_{\mu} \rightarrow \nabla_{\mu}=\partial_{\mu}-\Gamma_{\mu},
$$

where the curved-space Dirac matrices $\bar{\gamma}^{\mu}$ fulfill "local" commutation relations given in Eq. (15). These depend on the space-time coordinates. The spin-connection matrix $\Gamma_{\mu}$ describes the space-time curvature. The Dirac equation assumes the form

$$
\left(\mathrm{i} \bar{\gamma}^{\mu} \nabla_{\mu}-m_{I}\right) \psi=0
$$

under gravitational coupling. We recall that the free-space Dirac matrices fulfill

$$
\left\{\gamma^{\mu}, \gamma^{\nu}\right\}=2 g^{\mu \nu}=2 \operatorname{diag}(1,-1,-1,-1) .
$$

By contrast, the curved-space Dirac matrices fulfill

$$
\left\{\bar{\gamma}^{\mu}, \bar{\gamma}^{\nu}\right\}=2 \bar{g}^{\mu \nu}(x),
$$

where $\bar{g}^{\mu \nu}(x)$ is the metric of curved space-time. One $\operatorname{sets}^{27,30,33,34}$

$$
\bar{\gamma}^{\mu}=e_{A}^{\mu} \gamma^{A},
$$

where the $e_{A}^{\mu}$ are the coefficients of the vierbein or "tetrad," 33,34 and coefficients in the anholonomic basis are denoted by capital Latin letters $A, B, \ldots=0,1,2,3$. 
Roughly speaking, the derivation of the gravitational coupling proceeds as follows. As space-time is locally flat, one should be able to change the local Lorentz frames, and therefore, the vierbein coefficients, without changing the physics. By comparison, for quantum electrodynamics, $\frac{12}{2}$ one is able to change the local phase of the wave function independently at any point in space-time, provided one also performs a concomitant gauge transformation of the electromagnetic four-vector potentials.

On the basis of these considerations, one can derive a spinor representation of the local Lorentz group. One then formulates a covariant derivative with respect to the group $S O(1,3)$ of local Lorentz transformation, in accordance with the gauge principle. The paradigm is that the covariant derivative of the spinor-Lorentz transformation of the Dirac bispinor, formulated in the transformed coordinates, is equal to the spinor-local-Lorentz-transformation of the covariant derivative of the same Dirac bispinor. This can be formulated as follows:

$$
\nabla_{\mu}^{\prime} \psi^{\prime}=\left(\nabla_{\mu} \psi\right)^{\prime}, \quad \psi^{\prime}=S(\Lambda) \psi, \quad\left(\nabla_{\mu} \psi\right)^{\prime}=S(\Lambda) \nabla_{\mu} \psi
$$

A closer inspection ${ }^{32,40}$ of the problem reveals that this condition is fulfilled if one sets

$$
\Gamma_{\mu}=\frac{\mathrm{i}}{4} \omega_{\mu}^{A B} \sigma_{A B}, \quad \omega_{\mu}^{A B}=e_{\mu}^{A} \nabla_{\mu} e^{\nu B}
$$

where the Ricci rotation coefficients are denoted as $\omega_{\mu}^{A B}$, and the spin matrices are $\sigma_{A B}=\frac{\mathrm{i}}{2}\left[\gamma_{A}, \gamma_{B}\right]$. The covariant derivative acts as follows:

$$
\nabla_{\mu} e^{\nu B}=\partial_{\mu} e^{\nu B}+\Gamma_{\mu \rho}^{\nu} e^{\rho B} .
$$

Here, the $\Gamma_{\mu \rho}^{\nu}$ are the Christoffel symbols.

A change in the vierbein $e_{A}^{\mu} \rightarrow e_{A}^{\prime \mu}$ amounts to a local Lorentz transformation, and the $\nabla_{\mu}^{\prime}$ in Eq. (17) is understood as $\nabla_{\mu}^{\prime}=\partial_{\mu}-\Gamma_{\mu}^{\prime}$ (which entails no change in $\partial_{\mu}$, but a change in the Ricci rotation coefficients),

$$
\Gamma_{\mu}^{\prime}=\frac{\mathrm{i}}{4} \omega_{\mu}^{\prime A B} \sigma_{A B}, \quad \omega_{\mu}^{\prime A B}=e_{\mu}^{\prime A} \nabla_{\mu} e^{\nu B} .
$$

\section{Equivalence Principle for Antiparticles}

\subsection{Central-field problem}

The general formalism outlined in Sec. 2.2 has to be applied in practice. In a "weak" gravitational field described by a potential $\Phi$, one has the metric, ${ }^{24,41}$

$$
\begin{aligned}
\mathrm{d} s^{2} & =g_{\mu \nu} \mathrm{d} x^{\mu} \mathrm{d} x^{\nu}=(1+2 \Phi) \mathrm{d} t^{2}-(1-2 \Phi) \mathrm{d} \vec{r}^{2} \\
& =\left(1-\frac{2 G M}{r}\right) \mathrm{d} t^{2}-\left(1+\frac{2 G M}{r}\right) \mathrm{d} \vec{r}^{2},
\end{aligned}
$$


where in the case of a central gravitational potential, one has $\Phi=-G M / r$. An evaluation of the Christoffel symbols, and of the spin connection matrices, leads to the following result, ${ }^{20,24,41}$ which holds to first order in the gravitational coupling constant:

$$
\begin{aligned}
H_{G} & =\frac{1}{2}\{1+2 \Phi, \vec{\alpha} \cdot \vec{p}\}+\beta m_{I}(1+\Phi) \\
& =\frac{1}{2}\left\{1-\frac{2 G m M}{r}, \vec{\alpha} \cdot \vec{p}\right\}+\beta m_{I}\left(1-\frac{G M}{r}\right) .
\end{aligned}
$$

At the risk of oversimplification, we can say that the anticommutator term involving $\vec{\alpha} \cdot \vec{p}$ comes from the gravitational covariant derivative, while the term $\beta m(1-G m M / r)$ is due to the replacement of the flat-space Dirac matrices by their curved-space equivalents.

The functional form of the second term in Eq. (22) can be derived very easily by considering the Dirac equation for particles at rest, where there is no momentum operator at all. One applies the replacement (12) to the Dirac matrices, in the form $\gamma^{0} \rightarrow \bar{\gamma}^{0}$. The free Dirac equation for particles and antiparticles at rest is simply

$$
\mathrm{i} \gamma^{0} \partial_{t} \psi=m_{I} \psi \text {. }
$$

In curved space (central-field problem), one replaces

$$
\mathrm{i} \gamma^{0} \partial_{t} \psi=m_{I} \psi \rightarrow \mathrm{i} \bar{\gamma}^{0} \partial_{t} \psi=m_{I} \psi,
$$

where in view of Eq. (21),

$$
\bar{\gamma}^{0}=\sqrt{\bar{g}^{00}} \gamma^{0}=\sqrt{\frac{1}{1+2 \Phi}} \gamma^{0} \approx(1-\Phi) \gamma^{0}
$$

In the latter term, we have expanded the square root to first order in $\Phi$. One notes that $\bar{g}^{00}$ is a coefficient of the inverse metric $\bar{g}^{\mu \nu}$, where $\bar{g}^{\mu \nu} \bar{g}_{\nu \rho}=\delta^{\mu}{ }_{\rho}$. Hence, with $\bar{\gamma}_{0} \bar{\gamma}^{0}=1$, keeping track of upper and lower indices carefully, we have

$$
\mathrm{i} \partial_{t} \psi=m_{I} \bar{\gamma}_{0} \psi=\gamma^{0} m_{I}(1+\Phi) \psi=\beta m_{I}\left(1-\frac{G M}{r}\right) \psi .
$$

This simple consideration, at the risk of some over-simplification, rederives the second term on the right-hand side of Eq. (22). It means that the leading gravitational interaction term in a central field follows from the metric-induced modification of the Dirac $\gamma^{0}$ matrix alone, without any recourse to Christoffel symbols or gravitational connection matrices.

In order to derive the equivalence principle in leading order, it is sufficient to approximate Eq. (22) by

$$
H_{G}=\vec{\alpha} \cdot \vec{p}+\beta m_{I}(1+\Phi)=\vec{\alpha} \cdot \vec{p}+\beta m_{I}\left(1-\frac{G M}{r}\right),
$$

because the kinetic term already contains a momentum and is of higher order. 
If we add a nontrivial potential to the free Dirac Hamiltonian, as in Eq. (27), then it is not possible any more to diagonalize the Dirac Hamiltonian in bispinor space exactly. Rather, one employs a perturbative approach, with a FoldyWouthuysen transformation that eliminates the odd contributions, order-by-order in the momenta. ${ }^{12,38}$ A single step of the FW transformation, using [see Eq. (7)]

$$
U=\exp (\mathrm{i} S), \quad S=-\mathrm{i} \beta \frac{\vec{\alpha} \cdot \vec{p}}{2 m_{I}},
$$

then is sufficient to obtain the Foldy-Wouthuysen transformed, gravitationally coupled Hamiltonian

$$
H_{G}^{\mathrm{FW}}=U H_{G} U^{-1}=H_{G}+\mathrm{i}\left[S, H_{G}\right]+\frac{\mathrm{i}^{2}}{2 !}\left[S,\left[S, H_{G}\right]\right]+\cdots,
$$

which reads as follows:

$$
\begin{aligned}
H_{G}^{\mathrm{FW}} & =\beta\left[\frac{\vec{p}^{2}}{2 m_{I}}+m_{I} \Phi\right] \\
& =\left(\begin{array}{cc}
\left(\frac{\vec{p}^{2}}{2 m_{I}}-G \frac{m_{I} M}{r}\right) \mathbb{1}_{2 \times 2} & 0 \\
0 & -\left(\frac{\vec{p}^{2}}{2 m_{I}}-G \frac{m_{I} M}{r}\right) \mathbb{1}_{2 \times 2}
\end{array}\right) \\
& =\left(\begin{array}{cc}
H_{G}^{+} & 0 \\
0 & -H_{G}^{-}
\end{array}\right) .
\end{aligned}
$$

So, the particle and antiparticle Hamiltonians are equal,

$$
H_{G}^{+}=H_{G}^{-}=\left(\frac{\vec{p}^{2}}{2 m_{I}}-G \frac{m_{I} M}{r}\right) \mathbb{1}_{2 \times 2} .
$$

Note that the Newtonian central-field term $\left(-G m_{I} M / r\right)$ is obtained naturally in this Hamiltonian, for both particles as well as antiparticles. The emergence of the matrix $\mathbb{1}_{2 \times 2}$ implies that the energy is independent of the spin of the particle.

Let us remember that Newton stated that the property of a body called "mass" ("inertial mass") has to be proportional to the "weight" (which enters the gravitational force law), a principle otherwise known as the "weak equivalence principle" (WEP). Upon choosing the physical value of the gravitational constant $G$ appropriately, one finds the exact equivalence $m_{G}=m_{I}$ of the gravitational mass $m_{G}$ and the inertial mass $m_{I}$ of a particle. The EEP states that (i) WEP is valid, (ii) the outcome of any local nongravitational experiment is independent of the velocity of the freely-falling reference frame in which it is performed (local Lorentz invariance, LLI), and (iii) the outcome of any local nongravitational experiment is independent of where and when in the universe it is performed (local position invariance, LPI). 
We know that the classical Hamiltonian for a particle subject to a central gravitational potential is ${ }^{42}$

$$
H_{G, \mathrm{cl}}=\left(\frac{\vec{p}^{2}}{2 m_{I}}-G \frac{m_{G} M}{r}\right),
$$

where we neglect the spin (which does not exist in a classical theory), and denote the gravitational mass by $m_{G}$. By the correspondence principle, the quantum analogue is obtained from Eq. (32) by interpreting the momentum $\vec{p}$ in Eq. (32) as the momentum operator $\vec{p}=-\mathrm{i} \vec{\nabla}$.

A comparison of Eqs. (31) and (32) reveals that

$$
\begin{aligned}
& m_{G}=m_{I} \quad(\text { particles }), \\
& m_{G}=m_{I} \quad(\text { antiparticles }),
\end{aligned}
$$

thus establishing the equivalence principle in the Newtonian form, for antiparticles, at least within a leading-order calculation.

\subsection{Coupling to a general background}

We now investigate the general case, where the Dirac particle is not necessarily coupled to a static central field, but is coupled to a general (dynamic) background, with a (possibly time-dependent) space-time metric, which gives rise to time-dependent connection matrices $\Gamma_{\mu}$ [see Eq. (18)].

From nonrelativistic quantum mechanics, we know that the electromagnetic coupling can be described by the covariant coupling $\vec{p} \rightarrow \vec{p}-e \vec{A}$, where $\vec{A}$ is the vector potential of the electromagnetic field. The general electromagnetically and gravitationally coupled Dirac equation, for arbitrary electromagnetic four-vector potentials $A_{\mu}$, and arbitrary connection matrices $\Gamma_{\mu}$, reads as

$$
\left[\bar{\gamma}^{\mu}\left\{\mathrm{i}\left(\partial_{\mu}-\Gamma_{\mu}\right)-e A_{\mu}\right\}-m_{I}\right] \psi=0
$$

where the four-vector potential has components $A^{\mu}=(\Phi, \vec{A})$, and $e$ denotes the electron charge. We note the scalar potential $\Phi$ and the vector potential $\vec{A}$ (see Chap. 8 of Ref. 43). Recall the $\Gamma_{\mu}$ matrices from Eq. (18):

$$
\Gamma_{\mu}=\frac{\mathrm{i}}{4} \omega_{\mu}^{A B} \sigma_{A B}, \quad \omega_{\mu}^{A B}=e_{\mu}^{A} \nabla_{\mu} e^{\nu B}, \quad \sigma_{A B}=\frac{\mathrm{i}}{2}\left[\gamma_{A}, \gamma_{B}\right]
$$

Now, let us carry out the charge conjugation, which is tantamount to the particle $\rightarrow$ antiparticle transformation. Under transposition and complex conjugation, one obtains

$$
\psi^{+}\left(\left(\bar{\gamma}^{\mu}\right)^{+}\left(-\mathrm{i} \overleftarrow{\partial}_{\mu}-e A_{\mu}\right)+\mathrm{i} \Gamma_{\mu}^{+}\left(\bar{\gamma}^{\mu}\right)^{+}-m_{I}\right)=0
$$

where the differential operator acts on the $\psi$ to the left. We remember that $\Gamma_{\mu}$ is matrix-valued. The Hermitian adjoint $\Gamma_{\mu}^{+}$of $\Gamma_{\mu}$ is calculated as follows:

$$
\Gamma_{\mu}^{+}=-\frac{\mathrm{i}}{4} \omega_{\mu}^{A B} \sigma_{A B}^{+}=-\frac{\mathrm{i}}{4} \omega_{\mu}^{A B} \gamma^{0} \sigma_{A B} \gamma^{0}=-\gamma^{0} \Gamma_{\mu} \gamma^{0}
$$


where one has used the result

$$
\begin{aligned}
\sigma_{A B}^{+} & =-\frac{\mathrm{i}}{2}\left[\gamma_{B}^{+}, \gamma_{A}^{+}\right]=-\frac{\mathrm{i}}{2} \gamma^{0}\left[\gamma^{0} \gamma_{B}^{+} \gamma^{0}, \gamma^{0} \gamma_{A}^{+} \gamma^{0}\right] \gamma^{0} \\
& =-\frac{\mathrm{i}}{2} \gamma^{0}\left[\gamma_{B}, \gamma_{A}\right] \gamma^{0}=-\gamma^{0} \sigma_{B A} \gamma^{0}=\gamma^{0} \sigma_{A B} \gamma^{0}
\end{aligned}
$$

Here, we recall that we use the Dirac representation, given in Eq. (2), where one has the relationship $\gamma^{0}\left(\gamma^{A}\right)^{+} \gamma^{0}=\gamma^{A}$. One can immediately infer that $\gamma^{0}\left(\bar{\gamma}^{\mu}\right)^{+} \gamma^{0}=\bar{\gamma}^{\mu}$ (for the curved-space matrices, which are related to the flat-space $\gamma^{\mu}$ by the vierbein coeffcients $\left.e_{A}^{\mu}\right)$. An insertion of $\gamma^{0}$ matrices, using the identity $\left(\gamma^{0}\right)^{2}=1$, leads to

$$
\left(\psi^{+} \gamma^{0}\right) \gamma^{0}\left[\left(\bar{\gamma}^{\mu}\right)^{+}\left(-\mathrm{i} \overleftarrow{\partial}_{\mu}-e A_{\mu}\right)-\mathrm{i} \gamma^{0} \Gamma_{\mu} \gamma^{0}\left(\bar{\gamma}^{\mu}\right)^{+}-m_{I}\right] \gamma^{0}=0
$$

For absolute clarity, observe that the sign change in the prefactor multiplying the $\Gamma_{\mu}$ matrix, as compared to Eq. (36), is due to Eq. (37). This leads to the equation

$$
\bar{\psi}\left[\bar{\gamma}^{\mu}\left(-\mathrm{i} \overleftarrow{\partial}_{\mu}-e A_{\mu}\right)-\mathrm{i} \Gamma_{\mu} \bar{\gamma}^{\mu}-m_{I}\right]=0
$$

Under an additional transposition, one obtains

$$
\left[\left(\bar{\gamma}^{\mu}\right)^{\mathrm{T}}\left(-\mathrm{i} \partial_{\mu}-e A_{\mu}\right)-\mathrm{i}\left(\bar{\gamma}^{\mu}\right)^{\mathrm{T}} \Gamma_{\mu}^{\mathrm{T}}-m_{I}\right] \bar{\psi}^{\mathrm{T}}=0 .
$$

One introduces the charge conjugation matrix $C=\mathrm{i} \gamma^{2} \gamma^{0}$ (in the Dirac representation) with the defining property

$$
C\left(\gamma^{A}\right)^{\mathrm{T}} C^{-1}=-\gamma^{A}, \quad C\left(\bar{\gamma}^{\mu}\right)^{\mathrm{T}} C^{-1}=-\bar{\gamma}^{\mu},
$$

so that the charge conjugated spinor is $\psi^{\mathcal{C}}(x)=C \bar{\psi}^{\mathrm{T}}(x)$. In the Dirac representation, a possible choice is $C=\mathrm{i} \gamma^{2} \gamma^{0}$. Insertion of the identity matrix in the form $\mathbb{1}=C^{-1} C$ in Eq. (41) leads to

$$
\left[C\left(\bar{\gamma}^{\mu}\right)^{\mathrm{T}} C^{-1}\left(-\mathrm{i} \partial_{\mu}-e A_{\mu}\right)-\mathrm{i} C\left(\bar{\gamma}^{\mu}\right)^{\mathrm{T}} C^{-1} C \Gamma_{\mu}^{\mathrm{T}} C^{-1}-m_{I}\right] C \bar{\psi}^{\mathrm{T}}=0 .
$$

One calculates the identity

$$
\begin{aligned}
C \Gamma_{\mu}^{\mathrm{T}} C^{-1} & =\frac{\mathrm{i}}{4}\left\{\frac{\mathrm{i}}{2} \omega_{\mu}^{A B} C\left[\gamma_{B}^{\mathrm{T}}, \gamma_{A}^{\mathrm{T}}\right] C^{-1}\right\} \\
& =\frac{\mathrm{i}}{4}\left\{\frac{\mathrm{i}}{2} \omega_{\mu}^{A B}\left[-\gamma_{B},-\gamma_{A}\right]\right\}=-\Gamma_{\mu} .
\end{aligned}
$$

The charge conjugated (particle $\rightarrow$ antiparticle transformed) Dirac equation fulfilled by $\psi^{\mathcal{C}}(x)$ thus reads as

$$
\left[\left(-\bar{\gamma}^{\mu}\right)\left(-\mathrm{i} \partial_{\mu}-e A_{\mu}\right)-\mathrm{i} \bar{\gamma}^{\mu} \Gamma_{\mu}-m_{I}\right] \psi^{\mathcal{C}}(x)=0,
$$

or alternatively as

$$
\left[\bar{\gamma}^{\mu}\left\{\mathrm{i}\left(\partial_{\mu}-\Gamma_{\mu}\right)+e A_{\mu}\right\}-m_{I}\right] \psi^{\mathcal{C}}(x)=0 .
$$


The only difference between the original Dirac equation (34) and Eq. (46) is the sign of the physical charge $e$ of the particle. The sign and the functional form of the gravitational coupling term remains the same.

Note that $\psi^{\mathcal{C}}(x)=C \bar{\psi}^{\mathrm{T}}(x)=C\left(\psi^{+} \gamma^{0}\right)^{\mathrm{T}}(x)=C \gamma^{0} \psi^{*}(x)$ involves a complex conjugation. By way of example, let us assume that $\psi(x)$ is proportional to $\exp (-\mathrm{i} E t+\mathrm{i} \vec{k} \cdot \vec{r})$, with a positive energy eigenvalue $E>0$. In this case, $\psi^{*}(x)$ and, thus, $\psi^{\mathcal{C}}(x)$ will be proportional to $\exp (\mathrm{i} E t-\mathrm{i} \vec{k} \cdot \vec{r})$, with a negative eigenvalue of the time derivative operator $\mathrm{i} \partial_{t}$. The latter form is the characteristic dependence for an antiparticle wave function. Let us note that the comparison of Eqs. (34) and (46) reveals that the gravitational coupling term is invariant under the particle $\rightarrow$ antiparticle transformation in a general dynamic space-time background whose metric gives rise to Ricci rotation coefficients $\omega_{\mu}^{A B}$ and connection matrices $\Gamma_{\mu}$.

Furthermore, we note that both Eqs. (34) and (46) contain the inertial mass term $m_{I}$. In principle, neither of these equations say anything about the identification of the inertial with the gravitational mass. However, their comparison and the retention of the sign as well as the entire functional form of the gravitational coupling term in unchanged form under the particle $\rightarrow$ antiparticle transformation, shows that if the equivalence $m_{I}=m_{G}$ for antiparticles holds in one special example case (e.g. the central-field problem, see Sec. 2.2), then it must hold for any general space-time geometry (including all relativistic correction terms). Additional considerations regarding the relativistic terms, for the special case of a central-field problem, can be found in Ref. 19.

\section{Conclusions}

Let us briefly review the most important results of the current investigation. In Sec. 2, we present the formalism of gravitational coupling for spin- $1 / 2$ particles on which this paper is based. First, recall that the Dirac Hamiltonian describes particles and antiparticles simultaneously (Subsec. 2.1) and recover a few details on the free Dirac equation. The covariant coupling to curved space-time (gravitational coupling) is discussed in Subsec. 2.2. We then derive the equivalence principle for antiparticles in an illustrative way (in Sec. 3), based on the Foldy-Wouthuysen transformed Dirac Hamiltonian. In Subsec. 3.1, the central-field problem is investigated, and the derivation culminates in Eq. (31), where the particle and antiparticle Hamiltonians in a gravitational field are given. Illustrative remarks on the differences between the electrostatic and gravitational central-field problems are given in App. A. The derivation is generalized to arbitrary curved space-time backgrounds in Subsec. 3.2, for a general Dirac particle, coupled to both electromagnetic as well as gravitational fields. The electromagnetic coupling term reverses sign under the particle $\rightarrow$ antiparticle transformation, as it should, while the gravitational term retains its sign and its full functional form. The conclusions of Subsec. 2.2 imply that $m_{I}=m_{G}$ for the central-field problem ("matching"), and the considerations 
of Subsec. 3.2 generalize this finding to arbitrary (possibly dynamic) curved space times.

In summary, Sec. 3 is devoted to a derivation of the (weak) equivalence principle for antiparticles, showing the equivalence of the inertial and gravitational mass $m_{I}=m_{G}$ for antiparticles. Within the Dirac formalism, any deviation of the gravitational mass of antiparticles as compared to the gravitational mass of particles is thus constrained by the very precise available comparisons of the corresponding inertial masses, which are available for some elementary particles. ${ }^{35,36}$ Conversely, if a deviation of the gravitational mass of an antiparticle from that of a particle should be found in an upcoming experiment, then we would have a clear motivation for fundamentally changing our physical picture of the gravitational interaction in general - not only for antiparticles, but conceivably, also for particles.

This paper is rounded off by two more appendices. The first of these, App. B, is devoted to an illustration of the limitations of the Einstein equivalence principle, for both particles as well as antiparticles. As already mentioned, the Einstein equivalence principle states, among other postulates, that the outcome of any local nongravitational experiment is independent of where and when in the universe it is performed (local position invariance, LPI). One of the "compatibility issues" of this postulate, with respect to the uncertainty principle, is that, at some level of precision, one cannot tell any more with absolute certainty where precisely the experiment was performed, because of the nonvanishing positional uncertainty of the wave function. Another "incompatibility" of the separation of nongravitational and gravitational experiments is found when we consider that at some level of accuracy, one cannot separate a nongravitational from a gravitational experiment any more. Even the electron spin, which would flip in a transition from, say, an $n P_{1 / 2}$ to an $n P_{3 / 2}$ level in hydrogen, couples to gravity (here, $n$ is the principal quantum number). These observations lead to tiny deviation of atomic clock comparisons at different places in a gravitational potential, from the formula (B.1) that would otherwise be implied if the equivalence principle had no incompatibilities with quantum mechanics. Reassuringly, we can say that deviations from Eq. (B.1), given in Eq. (B.2), are numerically small. ${ }^{24}$

Finally, we also look at the Penrose conjecture in App. C, which is motivated by the observation that an "uncertain" quantum distribution of mass, as implied by the Heisenberg uncertainty relation, implies an uncertainty in the solution of the Einstein equations due to the quantum effects. The latter leads to an "uncertainty" in the determination of space-time curvature in the vicinity of the quantum mechanical wave function. This uncertainty can only be resolved once the wave function collapses. Our analysis, presented in App. C, allows us to interpret the Penrose conjecture as the "principle of reasonable determinability of the space-time metric, in view of the uncertainty of the mass distribution associated with any quantum mechanical wave packet." We find that the collapse times implied by the Penrose conjecture [see Eqs. (C.1) and (C.2)], for typical quantum systems, are longer than the age of the Universe. The good news derived from this observation is that, 
apparently, in practical applications, the collapse of the wave function, due to other physical phenomena, happens on such short time scales that the concomitant uncertainty in the determination of the space-time curvature around the massive, quantum mechanical objects, is negligible. If collapse always happens on time scales shorter than would be postulated in the framework of the Penrose conjecture, then the "principle of reasonable determinability of the space-time metric" is always fulfilled in practice - because of reasons that have nothing to do with gravity but with our apparent inability to produce coherent superpositions of macroscopic quantum objects.

\section{Acknowledgments}

This work was supported by the National Science Foundation (Grant PHY1710856). The author thanks G. Gabrielse, A. Geraci, T. Kovachy, M. Cavaglia, I. Nándori, J. H. Noble and M. Bojowald for useful discussions. Part of this work was completed during a visit to the Center for Fundamental Physics at Northwestern University in Chicago; the author is grateful to G. Gabrielse for the hospitality.

\section{Appendix A. (Naïve) Insertion of a Gravitational Potential}

Let us briefly review why it is not consistent to simply insert the gravitational potential into the Dirac Hamiltonian. This procedure would otherwise conceivably lead to a Hamiltonian of the functional form

$$
\vec{\alpha} \cdot \vec{p}+\beta m_{I}-G \frac{m_{G} M}{r},
$$

which differs from Eq. (27). One might be tempted to consider the Hamiltonian (A.1) because of its analogy to the Dirac-Coulomb Hamiltonian, which forms the basis for the description of the relativistic hydrogen atom. The latter Hamiltonian reads as

$$
H_{\mathrm{DC}}=\vec{\alpha} \cdot \vec{p}+\beta m_{I}-\frac{Z \alpha}{r},
$$

where $Z$ is the nuclear charge, and $\alpha$ is the fine-structure constant. 12 One immediate question which comes to mind is why one cannot simply insert the static gravitational potential into the Dirac Hamiltonian, in analogy to Eq. (A.1), as one would do for the Coulomb potential in Eq. (A.2). The answer is, in short, that the Coulomb potential insertion can be traced to the $U(1)$ covariant derivative, in the sense of the replacement $\mathrm{i} \partial_{\mu} \rightarrow \mathrm{i} \partial_{\mu}-e A_{\mu}$ [see Eqs. (A.3) and (A.4)], where $A_{\mu}$ is the external four-vector potential, but gravity is not a $U(1)$ gauge theory like quantum electrodynamics (QED).

In order to put things into perspective, we note that the Coulomb potential makes its way into the Dirac Hamiltonian by way of the covariant derivative, which amounts to a replacement of the partial derivative by the $U(1)$ covariant derivative

$$
\mathrm{i} \partial_{\mu} \rightarrow \mathrm{i} D_{\mu}=\mathrm{i} \partial_{\mu}-e A .
$$


For $\mu=0$ (timelike component), this implies that

$$
\mathrm{i} \partial_{t} \rightarrow \mathrm{i} \partial_{t}-e\left(\frac{(-Z e)}{4 \pi r}\right),
$$

where $Z$ is the nuclear charge number, and $e$ is the electron charge $\left(e^{2}=4 \pi \alpha\right.$ in natural units).

By contrast, note that the gravitational interaction is not based on a $U(1)$ gauge theory. A gauge transformation under gravitational coupling of a Dirac particle ensures the covariance with respect to local Lorentz transformations [gauge group $S O(1,3)]$, not $U(1)$ gauge transformations. ${ }^{25-30}$ For absolute clarity, we should remark that a Foldy-Wouthuysen transformation of the electrostatically coupled Hamiltonian (A.2) leads to two Hamiltonians, one for the particle, the other, for the antiparticle. These describe the behavior of the electron and positron, respectively, in a central binding Coulomb field. They constitute special cases of the more general Eq. (5) of Ref. 24. For the central-field gravitational problem, the starting point of the corresponding investigation has to be the Hamiltonian given in Eq. (22).

\section{Appendix B. Limitations of Einstein's Equivalence Principle}

After our intensive investigations of the relation of the equivalence principle to particle $\rightarrow$ antiparticle transformations, one might ask about further possible limitations to the general validity of Einstein's Equivalence Principle. In view of the relations derived in Subsec. 3.2 (particle-antiparticle symmetry), conceivable limitations would equally affect both particles and antiparticles. Roughly speaking, one might ask if a fully deterministic theory, like general relativity, could be fully compatible with a nondeterministic theory, like quantum mechanics, given that the latter has to accommodate Heisenberg's uncertainty principle.

As already mentioned, but recalled for convenience, the EEP states that (i) the outcome of any local nongravitational experiment is independent of the velocity of the freely-falling reference frame in which it is performed (local Lorentz invariance, LLI), and that, (ii) the outcome of any local nongravitational experiment is independent of where and when in the universe it is performed (local position invariance, LPI). The question, though, is whether or not we precisely know where and when in the Universe the experiment actually was performed, given the Heisenberg uncertainty principle. In particular, the nonvanishing positional uncertainty of the electron wave packet in an atom leads to an uncertainty in the exact position where, say, a spectroscopically measured quantum jump took place.

Roughly speaking, one can say that the validity of LPI is limited by the fact that, due to quantum mechanics, at some level of accuracy, one cannot separate a nongravitational experiment from a gravitational one. Let us illustrate this statement by way of example, following Ref. 24. Relativistic geodesy 44 is based on the comparison of the proper times $\mathrm{d} \tau_{1}$ and $\overline{\mathrm{d}} \tau_{2}$ of two atomic clocks located at gravitational 
potentials $\Phi_{1}$ and $\Phi_{2}$,

$$
\frac{\mathrm{d} \tau_{1}}{\mathrm{~d} \tau_{2}}=\frac{\sqrt{1+2 \Phi_{1}}}{\sqrt{1+2 \Phi_{2}}} .
$$

If the proper times measured by the atomic clocks follow the above relation, then full compatibility with the EEP, notably, LPI, is achieved.

According to Ref. 24, the deviations can be expressed as follows:

$$
\frac{\mathrm{d} \tau_{1}}{\mathrm{~d} \tau_{2}}=\frac{\sqrt{1+2 \Phi_{1}}+\left|\frac{\Phi_{1}}{\Phi_{0}}\right|^{n} C_{n}(M)}{\sqrt{1+2 \Phi_{2}}+\left|\frac{\Phi_{2}}{\Phi_{0}}\right|^{n} C_{n}(M)} .
$$

Here, $n$ refers to the power of the gravitational potential at which the respective correction term enters (no summation over $n$ !). In typical cases, one has either $n=2$ or $n=3$. The coefficients $C_{n}(M)$ depend on the mass of the gravitational center, and on the effect under study (power law coefficient $n$ ). The reference potential $\Phi_{0}$ is defined in Eq. (B.3). Some of the correction terms of the functional form $\left|\Phi_{1} / \Phi_{0}\right|^{n} C_{n}(M)$ are due to the fact that the quantum mechanical wave function involved in the measurement of the proper time intervals $\mathrm{d} \tau_{1}$ and $\mathrm{d} \tau_{2}$ "wiggles," so that it is no longer possible to pinpoint the exact location in the gravitational field where the measurement of the proper time interval took place. Put differently, the Heisenberg uncertainty principle implies that at some point, one cannot separate a gravitational from an electromagnetic experiment. This leads to deviations from the "perfect" scaling implied by Eq. (B.1).

We note that in Eqs. (74), (76) and (77) of Ref. 24, one should understand the factor $|\Phi|^{n}$ as $\left|\Phi / \Phi_{0}\right|^{n}$; the reference potential $\Phi_{0}$ used in Eq. (B.2) is

$$
\Phi_{0}=\frac{G M_{\oplus}}{R_{\oplus}},
$$

which is equal to the modulus of the gravitational potential on the Earth's surface. The corrections which lead to a deviation from the scaling (B.1), according to Eq. (B.2), have been analyzed in detail as $\delta E^{(\mathrm{i}) \cdots(\mathrm{iv})}$ in Ref. 24. A brief synopsis can be given as follows:

- $\delta E^{(\mathrm{i})}$ is the quadrupole term, evaluated for an excited atomic state (it leads to an effect which scales as $|\Phi|^{n=3}$ ).

- $\delta E^{(i i)}$ is a second-order effect due to the variation of the gravitational potential on distance scales commensurate with the size of the atom (it leads to an effect with $n=2$ ).

- $\delta E^{(\mathrm{iii})}$ is the so-called Fokker-Planck correction, which is caused by the coupling of the electron spin to the gravitational field (the power-law dependence has $n=3)$.

- Finally, $\delta E^{(\mathrm{iv})}$ is a first-order correction, due to the variation of the gravitational potential on a distance scale commensurate with the molecular wave function; it is nonvanishing only for oriented, diatomic molecules (the effect has $n=2$ ). 
We note that the corrections $\delta E^{(\mathrm{ii})}$ and $\delta E^{(\mathrm{iv})}$ depend on the quantum-mechanical positional uncertainty in the system and would thus vanish were it not for Heisenberg's uncertainty principle. Of these, as shown in Table 1 of Ref. $24, \delta E^{(\text {iv })}$ leads to coefficients in the range

$$
C_{2}^{(\mathrm{iv})}\left(M_{\oplus}\right) \sim 10^{-20} \cdots 10^{-18} \quad \text { (oriented molecules) }
$$

for $\delta E^{(i v)}$ (for the Earth's gravitational field). This effect could thus be measurable in the foreseeable future, as spectroscopic techniques approach the $10^{-19}$ precision level. 45

Corrections $\delta E^{(\mathrm{i})}, \delta E^{(\mathrm{ii})}$ and $\delta E^{(\mathrm{iv})}$, vanish in the hypothetical limit of a vanishing Bohr radius of the atom. One might ask if full compatibility with the EEP could be restored if we could hypothetically "switch off the Heisenberg principle." However, the correction $\delta E^{(\mathrm{iii})}$ comes into play, as a manifestation of the Fokker precession (FP) Hamiltonian, which, for an electron interacting with the gravitational field of the Earth, reads as

$$
H_{\mathrm{FP}}=\frac{3 G M_{\oplus}}{4 m_{e}} \frac{\vec{\sigma} \cdot \vec{L}}{R^{3}} .
$$

This Hamiltonian describes the coupling of the electron spin to the gravitational field of the Earth. It is interesting to note that it is proportional to the inverse of the mass of the electron. Let us suppose that we drive the $2 P_{1 / 2} \rightarrow 2 P_{3 / 2}$ spin-flip transition in atomic hydrogen with a laser, an experiment which, a priori, would be understood as a fundamentally nongravitational experiment. The Fokker precession Hamiltonian, which is the gravitational analogue of the Russell-Saunders spin-orbit coupling, constitutes a gravitational coupling term which cannot be "switched off" in nature, not even in the limit of a vanishing Bohr radius. Its presence illustrates the statement made above, which implies that at some level of precision, it might be in principle impossible to perform purely nongravitational experiments, because all particles involved in the experiments will also be subjected to other fundamental forces. Numerical estimates lead to the result that

$$
C_{3}^{(\mathrm{iii})}\left(M_{\oplus}\right) \sim 10^{-44} \quad \text { (atoms and molecules). }
$$

As such, the effect, while of utmost theoretical interest, will probably elude detection on atomic systems in the foreseeable future. The theoretical interest is enhanced by the fact that the Fokker-precession term is generated by noncommutativity of momentum operators and the gravitational potential, as a close inspection of its derivation $^{20}$ shows; i.e. it is a true quantum effect beyond the scaling of the proper time of the atomic clocks with $\sqrt{1+2 \Phi}$, which can be obtained if we ignore the quantum commutators. $\stackrel{24}{ }$

A further remark is in order. Here, as well as in Ref. 24, we have concentrated on effects which persist even at zero temperature. The first indication of a possible violation of the equivalence principle due to quantum effects was in fact mentioned in a series of papers ${ }^{46}-\underline{48}$ (see also Ref. 49 ), where the authors analyzed a possible 
violation of the Einstein equivalence principle at finite temperature, for an electron in contact with a heat bath of photons. The calculations reported in Refs. 46-49 are manifestly based on finite-temperature field theory; all effects considered in Refs. 46-49 vanish in the zero-temperature limit considered in this work.

Specifically, in Refs. 46-49, the gravitational mass of the particle at finite temperature is derived based on the $\mu=\nu=0$ component of the energy-momentum tensor $\tau^{\mu \nu}$, and the coupling to the gravitational field is described as in Eq. (16) of Ref. 49, being proportional to a term of the form $h_{\mu \nu} \tau^{\mu \nu}$ in an equation of the form (in our notation),

$$
\left(\mathrm{i} \gamma^{\mu} \partial_{\mu}-m_{I}\right) \psi=\frac{1}{2} h_{\mu \nu} \tau^{\mu \nu} \psi,
$$

where we assume vanishing temperature (hence, the vector $I_{\mu}$ in the notation of Ref. 49 vanishes), and $h_{\mu \nu}$ is taken as $h_{\mu \nu}=2 \Phi \operatorname{diag}(1,1,1,1)$ according to the text following Eq. (16) of Ref. 49, where $\Phi$ is the gravitational potential. It would be interesting to analyze if this formalism is equivalent to the covariant coupling discussed here in Sec. 2. Moreover, it would also be interesting to verify if the effects described in Refs. 46-49 can be rederived, e.g. for a central gravitational field, based on the approach described in Subsec. 3.1. Note that the leading gravitational coupling term written in Subsec. 3.1 is due to a simple mechanism which avoids the covariant derivative. Namely, it comes from the $\bar{\gamma}^{0}$ which is proportional to $1 / \sqrt{1+2 \Phi} \approx 1 /(1+\Phi)$ [see Eq. (25)]. The factor $1+\Phi$ then meanders into the numerator of the right-hand side of the Dirac equation, after solving for the time derivative operator, and multiplies the (entire) mass term, thus establishing the gravitational coupling in the central field [see Eq. (26) and pertinent remarks following the mentioned equation]. It would be extremely interesting to analyze, in detail, the relation of the conjectured temperature-dependent violation of the Einstein equivalence principle (see Refs. 46-49) to the formalism of the gravitationally coupled Dirac equation, laid out in this work. While further steps in this direction are beyond the scope of the current investigation, we contend ourselves with the notion that violations of the Einstein equivalence principle due to quantum effects (at finite temperature) have been discussed in the literature before.

\section{Appendix C. Relation of our Considerations to the Penrose Conjecture}

Another potential limitation to the applicability of EEP comes from the Penrose conjecture. $[0-52$ Roughly speaking, this conjecture deals with the following problem. Due to the Heisenberg principle, the precise location of a particle described by a quantum mechanical wave function is in principle endowed with uncertainty. That means that we have a physical situation where the exact shape of a mass distribution that needs to enter the Einstein equations is unknown (due to quantum uncertainty) unless the wave function has collapsed. Yet, at some point, we need to know where the particle is, or else we could not determine the space-time curvature (metric) 
around the objects. In order to ensure that the uncertainty in determining the metric remains does not grow without bound, one postulates that the wave function must collapse at some point, in a nonconstant gravitational field. This observation is the origin of the Penrose conjecture. $\underline{50}^{-52}$

The conjecture then implies that collapse of the wave function should occur on a time scale

$$
t_{C} \sim \frac{\hbar}{E_{G}}
$$

where $E_{G}$ is a measure of the gravitational energy contained in the un-collapsed wave function. Various forms of $E_{G}$ have been discussed in the literature. ${ }^{24,50-54}$ An (unnumbered) equation on p. 595 of Ref. 50 puts

$$
E_{G}=-G \int \mathrm{d}^{3} x \int \mathrm{d}^{3} y \frac{\left[\rho(\vec{x})-\rho^{\prime}(\vec{x})\right]\left[\rho(\vec{y})-\rho^{\prime}(\vec{y})\right]}{|\vec{x}-\vec{y}|},
$$

where $\rho(\vec{r})$ and $\rho^{\prime}(\vec{r})$ are the two mass distributions, which represent possible outcomes of measurements of the position of the particle, after the wave function collapses. Equation (C.2) describes the gravitational self-energy of the difference between the two mass distributions.

Let us perform some order-of-magnitude estimates, writing

$$
\begin{aligned}
& E_{G}=-E_{S}-E_{S}^{\prime}+E_{I}, \\
& E_{S}=G \int \mathrm{d}^{3} x \int \mathrm{d}^{3} y \frac{\rho(\vec{x}) \rho(\vec{y})}{|\vec{x}-\vec{y}|}, \\
& E_{S}^{\prime}=G \int \mathrm{d}^{3} x \int \mathrm{d}^{3} y \frac{\rho^{\prime}(\vec{x}) \rho^{\prime}(\vec{y})}{|\vec{x}-\vec{y}|}, \\
& E_{I}=2 G \int \mathrm{d}^{3} x \int \mathrm{d}^{3} y \frac{\rho(\vec{x}) \rho^{\prime}(\vec{y})}{|\vec{x}-\vec{y}|} .
\end{aligned}
$$

Here, $E_{I}$ has the interpretation of a gravitational interaction energy between the two mass distributions, while $E_{S}$ and $E_{S}^{\prime}$ are the gravitational self-energies.

Order-of-magnitude estimates for typical quantum objects can be given as follows:

- The mass $\int \mathrm{d}^{3} x \rho(\vec{x}) \sim m$ is of the order of the mass of an atomic nucleus, or, the proton mass of $\sim 10^{-27} \mathrm{~kg}$.

- The typical distance $|\vec{x}-\vec{y}|$ in the self-energy integrals cannot be smaller than a de Broglie wavelength of the wave packet, or, the size of an atom, which is the Bohr radius $\sim\left(10^{-11} \cdots 10^{-10}\right) \mathrm{m}$.

- The typical distance $|\vec{x}-\vec{y}|$ in the interaction integrals cannot be smaller than the dimensions of technical device with which the atoms are being controlled, i.e. not smaller than a few nanometers, which is commensurate with today's microprocessor manufacturing standards, $\sim 10^{-9} \mathrm{~m}$. 
Hence, for typical quantum systems, all three entries in Eq. (C.3a) are of the order of, or smaller, than

$$
E_{G} \sim \frac{10^{-11} \times\left(10^{-27}\right)^{2}}{10^{-11}}=10^{54} \mathrm{~J} .
$$

For more detailed calculation based on the parameters of the Colella-OverhauserWerner experiment, ${ }^{55-58}$ see Ref. 24. In the experiments ${ }^{55-58}$ a quantum wave packet is split in an interferometer on macroscopic distance scales in a gravitational field (for recently enhanced versions, which rely on atomic rather than neutron interferometry, see Refs. 59 and 60). The mass distributions $\rho$ and $\rho^{\prime}$ correspond to the two arms of the interferometer. An important observation, compatible with the considerations above, is that the distance scale $|\vec{x}-\vec{y}|$, which enters the self-energy integrals $E_{S}$ and $E_{S^{\prime}}$, is typically smaller than those which enter the interaction integrals. Hence, the expression for $E_{G}$ given in Eq. (C.3a) comes out as negative for typical configurations; one might have to eliminate the minus sign in Eq. (C.2) or consider the modulus of the given quantity instead, in order to obtain a positive value for the collapse time $t_{C}$.

On account of the smallness of the reduced Planck constant, $\hbar \sim 10^{-34}$ Js, we have for typical quantum systems, in view of Eq. (C.4),

$$
t_{C} \sim 10^{20} \mathrm{~s},
$$

which is longer than the age of the Universe.

These estimates also imply that gravitationally induced wave collapse, according to the Penrose conjecture, does not lead to limitations for the functionality of quantum computers: Even if we control on the order of $n=10^{10}$ atoms coherently on microscopic dimension, the estimate given in Eq. (C.5) would be reduced by a factor $1 / n^{2} \sim 10^{-20}$, and still could not "collapse" the wave function on a time scale less than a second.

\section{References}

1. ALPHA Collab., Antihydrogen laser physics apparatus, see http://alpha-new.web. cern.ch.

2. ALPHA Collab. (C. Amole et al.), Nature (London) 483, 439 (2012).

3. ALPHA Collab. (C. Amole et al.), Nat. Commun. 4, 1785 (2013).

4. ATHENA Collab., ATHENA antihydrogen apparatus, see http://athena.web.cern. $\operatorname{ch} /$.

5. ATRAP Collab., Antihydrogen trap, see http://home.cern/science/experiments/ atrap.

6. ATRAP Collab. (G. Gabrielse et al.), Phys. Rev. Lett. 100, 113001 (2008).

7. AEGIS Proto-Collab. (A. Kellerbauer et al.), Nucl. Instrum. Methods Phys. Res. B 266, 351 (2008).

8. AGE Collab. (A. D. Cronin et al.), Letter of Intent: Antimatter gravity experiment (AGE) at Fermilab, available at: http://www.fnal.gov/directorate/program_ planning/Mar2009PACPublic/AGELOIFeb2009.pdf; see also http://www.phy.duke. edu/ phillips/gravity/frameIndex.html. 
9. P. J. Mohr, G. Plunien and G. Soff, Phys. Rep. 293, 227 (1998).

10. P. A. M. Dirac, Proc. R. Soc. London Ser. A 117, 610 (1928).

11. P. A. M. Dirac, Proc. R. Soc. London Ser. A 118, 351 (1928).

12. C. Itzykson and J. B. Zuber, Quantum Field Theory (McGraw-Hill, New York, 1980).

13. C. D. Anderson, Phys. Rev. 43, 491 (1933).

14. J. Maruani, J. Chin. Chem. Soc. 63, 33 (2016).

15. M. Kowitt, Int. J. Theor. Phys. 35, 605 (1996).

16. L. L. Foldy and S. A. Wouthuysen, Phys. Rev. 78, 29 (1950).

17. U. D. Jentschura and B. J. Wundt, Eur. Phys. J. C 72, 1894 (2012).

18. H. A. Bethe, Phys. Rev. 72, 339 (1947).

19. U. D. Jentschura, Phys. Rev. A 87, 032101 (2013) [Erratum: ibid. 87, 069903 (2013)].

20. U. D. Jentschura and J. H. Noble, Phys. Rev. A 88, 022121 (2013).

21. U. D. Jentschura and J. H. Noble, J. Phys. A 47, 045402 (2014).

22. J. H. Noble and U. D. Jentschura, Phys. Rev. A 92, 012101 (2015).

23. J. H. Noble and U. D. Jentschura, Phys. Rev. A 93, 032108 (2016).

24. U. D. Jentschura, Phys. Rev. A 98, 032508 (2018).

25. H. Tetrode, Z. Phys. 50, 336 (1928).

26. V. Fock and D. Iwanenko, Z. Phys. 56, 798 (1929).

27. V. Fock, Z. Phys. 57, 261 (1929).

28. V. Fock and D. Ivanenko, C.R. Acad. Sci. Paris 188, 1470 (1929).

29. H. Weyl, Proc. Natl. Acad. Sci. USA 15, 323 (1929).

30. D. R. Brill and J. A. Wheeler, Rev. Mod. Phys. 29, 465 (1957).

31. A. S. Blum, Quantum Gravity in the First Half of the Twentieth Century, eds. A. S. Blum and D. Rickles, Edition Open Sources (Max-Planck-Institute for the History of Science, 2018), pp. 49-56.

32. M. Bojowald, Canonical Gravity and Applications (Cambridge University Press, Cambridge, 2011).

33. O. S. Ivanitskaya, Lorentzian Basis and Gravitational Effects in Einstein's Theory of Gravity (in Russian) (Nauka i Technika, Minsk, USSR, 1969).

34. O. S. Ivanitskaya, N. V. Mitskievic and Y. S. Vladimirov, Proc. 114th Symp. of the Int. Astronomical Union, Leningrad, USSR, May 1985, eds. J. Kovelevsky and V. A. Brumberg (Kluwer, Dordrecht, 1985), pp. 177-186.

35. G. Gabrielse, A. Khabbaz, D. S. Hall, C. Heimann, H. Kalinowsky and W. Jhe, Phys. Rev. Lett. 82, 3198 (1999).

36. S. Ulmer, C. Smorra, A. Mooser, K. Franke, H. Nagaharma, G. Schneider, T. Higuchi, S. Van Grop, K. Blaum, Y. Matsuda, W. Quint, J. Walz and Y. Yamazaki, Nature (London) 524, 196 (2015).

37. U. D. Jentschura, Eur. Phys. J. D 61, 7 (2011).

38. J. D. Bjorken and S. D. Drell, Relativistic Quantum Mechanics (McGraw-Hill, New York, 1964).

39. J. D. Bjorken and S. D. Drell, Relativistic Quantum Fields (McGraw-Hill, New York, 1965).

40. U. D. Jentschura and G. S. Adkins, Quantum Electrodynamics and Beyond: Relativity, Atoms, Lasers and Gravity (World Scientific, Singapore, 2019).

41. C. M. Will, Phys. Rev. D 10, 2330 (1974).

42. H. Goldstein, Klassische Mechanik (Akademische Verlagsgesellschaft, Frankfurt am Main, 1963).

43. U. D. Jentschura, Advanced Classical Electrodynamics: Green Functions, Regularizations, Multipole Decompositions (World Scientific, Singapore, 2017). 
44. E. Mai and J. Müller, $Z F V-Z$. Geodäsie Geoinform. Landmanagement 138, 255 (2013).

45. K. Predehl, G. Grosche, S. F. F. Raupach, S. Droste, O. Terra, J. Alnis, T. Legero, T. W. Hänsch, T. Udem, R. Holzwarth and H. Schnatz, Science 336, 441 (2013).

46. J. F. Donoghue, B. R. Holstein and R. W. Robinett, Phys. Rev. D 30, 2561 (1984).

47. J. F. Donoghue, B. R. Holstein and R. W. Robinett, Phys. Rev. D 34, 1208 (1986).

48. J. F. Donoghue and B. R. Holstein, Eur. J. Phys. 8, 105 (1987).

49. M. Blasone, S. Capozziello, G. Lambiase and L. Petruzziello, Eur. Phys. J. Plus 134, 169 (2019).

50. R. Penrose, Gen. Relat. Gravit. 28, 581 (1996).

51. R. Penrose, Philos. Trans. R. Soc. London A 356, 1927 (1998).

52. R. Penrose, Found. Phys. 44, 557 (2014).

53. L. Diósi, Phys. Lett. A 120, 377 (1987).

54. L. Diósi, Phys. Rev. A 40, 1165 (1989).

55. A. W. Overhauser and R. Colella, Phys. Rev. Lett. 33, 1237 (1974).

56. R. Colella, A. W. Overhauser and S. A. Werner, Phys. Rev. Lett. 34, 1472 (1975).

57. U. Bonse and T. Wroblewski, Phys. Rev. Lett. 51, 1401 (1983).

58. U. Bonse and T. Wroblewski, Phys. Rev. D 30, 1214 (1984).

59. P. Asenbaum, C. Overstreet, T. Kovachy, D. D. Brown, J. M. Hogan and M. A. Kasevich, Phys. Rev. Lett. 118, 183602 (2017).

60. C. Overstreet, P. Asenbaum, T. Kovachy, R. Notermans, J. M. Hogan and M. A. Kasevich, Phys. Rev. Lett. 120, 183604 (2018). 